\title{
Simulation of steep waves interacting with a cylinder by coupling CFD and Lagrangian models.
}

\author{
Pablo Higuera $^{1}$, Eugeny Buldakov ${ }^{2}$, Dimitris Stagonas ${ }^{3}$ \\ ${ }^{1}$ University of Auckland, Department of Civil and Environmental Engineering, Private Bag 92019, Auckland, 1142 \\ ${ }^{2}$ University College London, Department of Civil Engineering, Gower Street, London, WC1E 6BT \\ ${ }^{3}$ Cranfield University, Centre for Thermal Energy Systems and Materials, College Rd, Cranfield, Bedford, MK43 0AL
}

\begin{abstract}
This paper presents numerical modelling results of the interaction between a group of steep waves and a fixed vertical cylinder performed with a one-way coupled hybrid model. A set of experimental data is used to benchmark the accuracy of the modelling results. The wavemaker signal generated in the physical experiments is used to reproduce the incident wave conditions without a priori knowledge of the rest of the dataset. A Lagrangian numerical wave flume propagates the wave group, producing the non-linear free surface elevation and wave kinematics with high accuracy in the vicinity of the cylindrical structure. This set of data is used as the input to the olaFlow CFD model, which calculates the wave-structure interaction on a small computational domain. One-way coupling approaches based on boundary conditions and relaxation zones are tested and compared in terms of the recorded free surface elevation and pressures at the structure. Results present an adequate degree of accordance and turbulence effects are found to be negligible in the simulations.
\end{abstract}

KEY WORDS: Wave-structure interaction; cylinder; Lagrangian wave model; olaFlow; OpenFOAM.

\section{INTRODUCTION}

Wave and structure interaction (WSI) is a field in which numerical modelling is nowadays being applied consistently and with increasing popularity. The challenges derived from modelling the impact of waves and structures are numerous. Marine and offshore structures are usually located in areas subjected to extremely harsh conditions, in which often waves present three-dimensional and highly-nonlinear processes such as wave breaking and wave impacts may result in impulsive loading.

Under such conditions the relevance of numerical modelling, and especially computational fluid dynamics (CFD), derives from the low number of underlying assumptions that the Navier-Stokes equations involve, from their inherent nonlinearity and from their capability to include turbulence dissipation effects via Reynolds-Averaged Navier-
Stokes (RANS) or Large Eddy Simulation (LES) turbulence models. Furthermore, despite its advantage and flexibility, numerical modelling results alone have a limited practical credibility if they are not accompanied by a reasonable validation against experimental measurements, which is why physical and numerical modelling should be deemed as complementary approaches.

Another of the limitations of CFD is the large computational cost associated with the simulations, which makes them an impractical approach to simulate extensive domains and long time series. Instead, hybrid modelling (HM) appears to be gaining momentum to reduce such limitations. The concept behind HM consists in simulating the different areas of interest with several numerical techniques, according to the complexity of the processes occurring within each one. For example, under certain circumstances wave propagation can be accurately simulated with a potential flow theory model instead of using CFD, thus saving a significant amount of computational resources and time. The potential flow theory modelling may be performed until close to the structure of interest, where the CFD model calculations would take over to simulate the detailed interactions (e.g. Lachaume et al. (2003); Kim et al. (2010); Guo et al. (2012)).

There are two principal approaches to link the numerical models. In oneway coupling, one of the models is run first independently from the other and the data is passed to the second one, so there is no connection of feedback loop between both models. In two-way coupling, both models are run concurrently and they exchange information at the interface (which can be a boundary or a region in space). This way, waves could ideally propagate across models seamlessly. Despite being a more complete and realistic approach, two-way coupling models present significant technical and numerical implementation challenges, for example, in terms of blending solutions from two different sets of equations.

In this paper we introduce a one-way coupling hybrid modelling approach comprised by a Lagangian and CFD models. The displacement of the physical wavemaker is the only input required by the Lagrangian model to propagate the wave group and generate the dataset of wave kinematics to feed the CFD model. Then the CFD model reproduces the incident wave conditions via a boundary condition and a relaxation zone 


\begin{tabular}{llllllll} 
& WP1 & WP2 & WP3 & WP4 & WP5 & WP6 & WP7 \\
\hline$x$ & 4.975 & 13.928 & 14.178 & 14.428 & 24.31 & 24.88 & 25.585 \\
$y$ & 0 & 0 & 0 & 0 & 0.275 & 0.275 & 0.275
\end{tabular}

Table 1 Location of the free surface elevation probes. $x$ measured from the wavemaker and $y$ measured from the flume centreline. Distances in $\mathrm{m}$.

\begin{tabular}{lllllllll} 
& PP1 & PP2 & PP3 & PP4 & PP5 & PP6 & PP7 & PP8 \\
\hline$z$ & 0.415 & 0.515 & 0.615 & 0.715 & 0.815 & 0.615 & 0.615 & 0.615 \\
$\beta$ & $0^{\circ}$ & $0^{\circ}$ & $0^{\circ}$ & $0^{\circ}$ & $0^{\circ}$ & $20^{\circ}$ & $90^{\circ}$ & $180^{\circ}$
\end{tabular}

Table 2 Location of the pressure probes. $z$ measured from the bottom of the flume and $\beta$ measured from the negative $x$ direction. Distances in $\mathrm{m}$, angles in degrees.

without requiring any tuning.

This paper is structured as follows. First, the physical experiments are described after this introductory chapter. The Lagrangian model is introduced next, and the wave propagation simulations to obtain the incoming wave conditions near the cylinder are validated. Afterwards, the CFD model is described, along with the different setups tested in twoand three-dimensions. Then, the comparison between the results from the CFD modelling and the experiments are analysed. Finally, the conclusions and future work are drawn.

\section{PHYSICAL EXPERIMENTS SETUP}

The experiments reproduced in this paper were performed in a flume $(110 \times 2.2 \times 2.0 \mathrm{~m})$ at the Franzius-Institute Laboratory in Hannover. The description of the flume and methodology of wave generation used in the experiments can be found in Sriram et al. (2015). The setup consisted in an instrumented fixed cylinder, with a diameter of $0.22 \mathrm{~m}$, subjected to the impact of focussed wave groups.

The cylinder centre was located $24.88 \mathrm{~m}$ from the wavemaker and 1.085 $\mathrm{m}$ from the sidewall of the flume, therefore it was not located exactly at the centreline of the flume due to a small deviation of $2.5 \mathrm{~cm}$. However, this distance has been deemed small enough as compared to the total width of the flume $(2.2 \mathrm{~m})$ to consider the case symmetric when later reproducing it numerically.

The wave conditions in the case reproduced in this work correspond to a uni-directional focused wave group with a constant steepness spectrum created by 32 components with frequencies between $0.34 \mathrm{~Hz}$ an 1.02 $\mathrm{Hz}$. The nominal focusing position is $x=23 \mathrm{~m}$ and the maximal wave amplitude is about $0.2 \mathrm{~m}$, for a working water depth of $0.7 \mathrm{~m}$. The focused waves were generated by a hydraulic piston wavemaker using the second order correction technique introduced in Schäffer (1993) and the time series of the wavemaker displacement has been provided at $100 \mathrm{~Hz}$ sampling frequency.

Free surface elevation (FSE) was sampled at $100 \mathrm{~Hz}$ at 7 different locations along the flume, as indicated in Table 1. A total of 8 pressure probes were mounted on the cylinder at the positions indicated in Table 2. Pressure sampling took place at 9,600 Hz, although the final signal has been downsampled to $100 \mathrm{~Hz}$.

\section{INCOMING WAVE CONDITIONS}

In this work we use a Lagrangian Numerical Wave Tank (LNWT), also previously used in Higuera et al. (2018), as a fast component of the hybrid model to reconstruct the incoming wave conditions. Full details of the numerical method used by the LNWT can be found in Buldakov et al. (2019).

The LNWT has been used to replicate the wave flume in the FranziusInstitute Laboratory in Hannover, where the experimental dataset was generated. The two-dimensional rectangular Lagrangian computational domain has a depth of $0.7 \mathrm{~m}$ and a length of $60 \mathrm{~m}$. The Lagrangian model is expected to reproduce the wave behaviour before the cylinder position $(x \approx 25 \mathrm{~m}$ ), therefore, the length of the computational domain is much shorter than the length of the experimental tank $(110 \mathrm{~m})$. Moreover, since the Lagrangian modelling is $2 \mathrm{D}$, the cylinder has not been reproduced.

A piston-type wavemaker has been modelled as a vertical wall moving with a prescribed displacement starting from the initial position $x=0$. The incoming wave for the experimental case was generated using the actual motion of the experimental wave paddle provided by the Comparative Study organisers. Unlike the cases presented in Higuera et al. (2018), in which no experimental wave generator movement was available, wave generation was straightforward in this work and no iteration procedure was required. A dissipative region has been implemented between $x=40 \mathrm{~m}$ and $x=60 \mathrm{~m}$ to reduce reflections from the far end of the Lagrangian numerical tank, where an impenetrability (slip) boundary condition is applied on the vertical end wall.

The computational parameters of the Lagrangian model have been selected as the result of a convergence test. The computations were carried out as the combination of three sizes of computational mesh $401 \times 11$, $601 \times 11$ and $801 \times 11$, and two time steps $0.004 \mathrm{sec}$ and $0.002 \mathrm{sec}$. The FSE was sampled at selected locations along the numerical wave tank corresponding to the positions of experimental wave probes (Table 1). The differences between the wave profiles far from the wave maker (WP7) were found negligible for both time steps and for the two largest horizontal mesh resolutions.

Buldakov et al. (2019) showed that the vertical mesh resolution has little effect on the FSE profile, but can have a significant impact on wavegenerated velocities. The convergence of velocity profiles for increased vertical mesh resolution was checked by an additional case with $601 \times 16$ mesh and $0.004 \mathrm{sec}$ time step. Slight differences between the wave profiles calculated with different vertical resolutions were observed, therefore, we selected the case with $601 \times 16$ mesh and $0.004 \mathrm{sec}$ time step as the main computational case. The computational time for modelling $60 \mathrm{sec}$ of wave evolution in serial with the selected mesh and time step was approximately 90 hours.

It should be noted that due to the implicit time marching scheme used by the Lagrangian solver the computational time grows rapidly with increasing mesh sizes. In this work the computational parameters are chosen to provide the optimum quality for wave kinematics. If the computational time was a limiting criterion, a case with a $601 \times 11$ mesh would provide an acceptable accuracy with considerably lower computation time ( 28 hours on a single processor for a $60 \mathrm{sec}$ run). For future work, improving the computational efficiency of the Lagrangian solver might be necessary. Some ways of achieving this are discussed in Buldakov et al. (2019).

As it can be observed in Figure 1, the Lagrangian numerical wave model reproduces the experimental FSE with an acceptable accuracy. Slight discrepancies could be caused by the reflection of the waves at the cylinder during the experiment and by the reflections of long wave compo- 


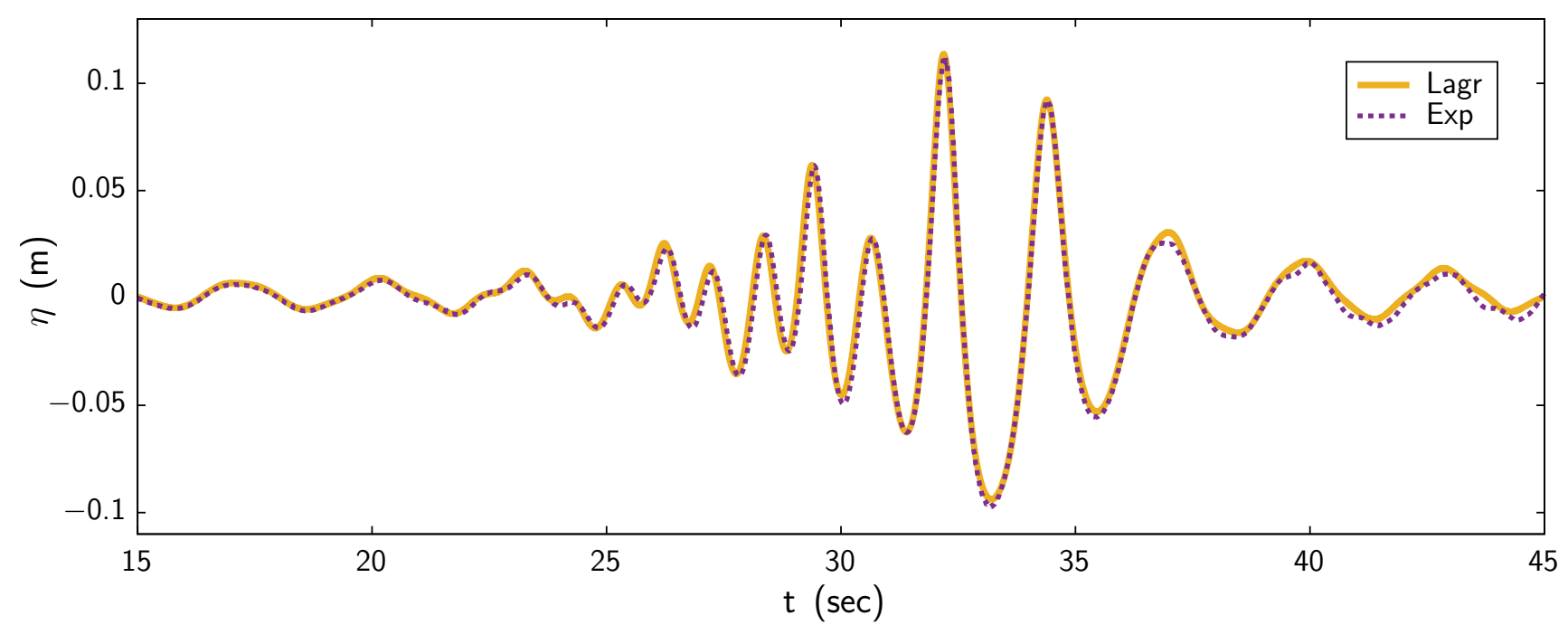

Fig. 1 Comparison of surface elevation of the experimental incoming wave at $x=14.428 \mathrm{~m}$ (WP4) with calculations by the Lagrangian model.

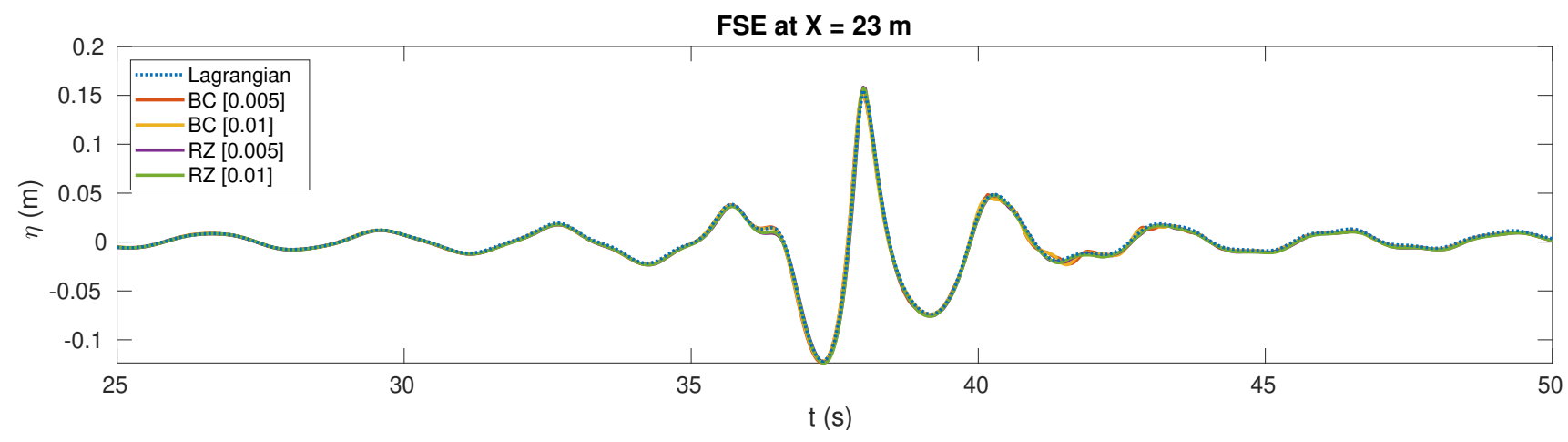

Fig. 2 Preliminary comparison of FSE between the Lagrangian and the 2D CFD simulations at $x=23 \mathrm{~m}$. Cell resolution given in $\mathrm{m}$.

nents not fully absorbed by the dissipative region at the far end of the Lagrangian numerical tank.

Wave kinematics (FSE and velocities) calculated by the Lagrangian model have been extracted between $x=23 \mathrm{~m}$ and $x=23.2 \mathrm{~m}$ to be used for the inlet relaxation zone and boundary condition for the CFD model.

\section{CFD MODEL AND SETUP}

The CFD solver olaFlow (Higuera et al., 2013; Higuera, 2017), developed with the OpenFOAM ${ }^{\circledR}$ (Weller et al., 1998) library has been applied in this work. olaFlow is a numerical model highly specialized in the simulation of waves and wave-structure interaction, solving the Reynolds-Averaged Navier-Stokes equations for two phases (water and air). The incompressible continuity and Navier-Stokes equations solved by olaFlow are:

$\nabla \cdot(\rho \mathbf{U})=\mathbf{0}$,

$\frac{\partial \rho \mathbf{U}}{\partial t}+\nabla \cdot(\rho \mathbf{U U})=$

$$
-\nabla p^{*}-\mathbf{g} \cdot \mathbf{r} \nabla \rho+\nabla \cdot\left(\mu \nabla \mathbf{U}-\rho \overline{\mathbf{U}^{\prime} \mathbf{U}^{\prime}}\right)+\sigma \kappa \nabla \alpha,
$$

in which $\rho$ is the density, $\mathbf{U}$ is the velocity vector, $t$ is time and $\nabla$ is the gradient operator. The dynamic pressure $\left(p^{*}\right)$ is derived from total pressure $(p)$ by the expression $p^{*}=p-\rho \mathbf{g} \cdot \mathbf{r}$, in which $\mathbf{g}$ is the acceleration due to gravity and $\mathbf{r}$ is the position vector. The fluid viscosity is included in the equations, where $\mu$ represents the molecular dynamic viscosity and $\rho \overline{\mathbf{U}^{\prime} \mathbf{U}^{\prime}}$ denotes the Reynolds stresses. The last term introduces the surface tension force, where $\sigma$ is the surface tension coefficient; $\kappa$ is the curvature of the free surface, calculated as $\nabla \cdot \frac{\nabla \alpha}{|\nabla \alpha|}$. Here, the Volume of Fluid (VOF) indicator function $(\alpha)$ tracks the amount of water per unit volume in the mesh cells.

In the VOF technique (Berberovic et al., 2009), the flow phases are advected as follows:

$\frac{\partial \alpha}{\partial t}+\nabla \cdot(\alpha \mathbf{U})+\nabla \cdot\left[\alpha(\mathbf{1}-\alpha) \mathbf{U}_{\mathbf{c}}\right]=\mathbf{0}$,

where $\mathbf{U}_{\mathbf{c}}$ is a numerical compression velocity to prevent the diffusion of the air-water interface.

The implementation of the one-way coupling interface between the Lagrangian model and olaFlow via a boundary condition (BC) has been previously reported in Higuera et al. (2018). This technique is linked with active wave absorption (Higuera, 2020), to allow dissipating the waves incident to the boundary (e.g. reflections on the cylinder) while still generating the target wave conditions. In this work two new enhancements have been introduced to the coupling interface.

Firstly, a non-uniform time series, having larger time steps $(0.1 \mathrm{~s})$ during time lapses of low free surface elevation gradients and smaller time steps $(0.01 \mathrm{~s})$ for steeper waves, has been produced with the Lagrangian model. This procedure ensures that the wave crests and troughs are generated more accurately, avoiding trimming them as a result of the linear interpolation in time used in the $\mathrm{BC}$, while also minimising the amount 
of input data needed to run the case.

Secondly, a relaxation zone (RZ) module has been programmed to relax the Lagrangian model kinematics (free surface elevation and velocities) and the Navier-Stokes equations solution at the wave generation zone. This technique requires additional input data, i.e. the wave kinematics within a zone in the vicinity of the wave generation boundary; then olaFlow uses bilinear interpolation (in time and space) to transfer the variables to each cell. Relaxation zones have been empirically observed to allow generating steeper waves without premature breaking at the wave generation boundary (although this is not an issue in the present simulations) and, most importantly, to absorb incoming waves (i.e., reflected from the cylinder) with a high degree of efficiency. However, it must be remarked that the computational times increase approximately by $5 \%$ to $20 \%$ when including RZs in the simulations, depending on their layout.

The RZ formulation chosen is the same previously applied in Higuera (2020), and introduced in Fuhrman et al. (2006); Jacobsen et al. (2012), which uses the equation

$\Lambda=w_{R} \Lambda_{\mathrm{NS}}+\left(1-w_{R}\right) \Lambda_{\mathrm{LA}}$

to relax the indicator function $(\alpha)$ and velocity vector $(\mathbf{U})$ explicitly after solving the VOF advection equation and before solving the pressure equation. Here, $\Lambda$ is the variable of interest and the subscripts $N S$ and $L A$ indicate the Navier-Stokes solution and the Lagrangian model values. The variable $w_{R}$ is a blending function, which varies smoothly between 0 and 1 within the RZ, following Fuhrman et al. (2006):

$w_{R}=1-\frac{e^{\sigma^{P}}-1}{e-1}$

where $\sigma$ is a coordinate system in which $w_{R}(\sigma=0)=1$ at the interface between the RZ and the inner domain and $w_{R}(\sigma=1)=0$ at the wave generation/absorption boundary. The parameter $P=3.5$ defines the shape of the relaxation function (Jacobsen et al., 2012).

The CFD modelling simulations involved two stages, neither of which required any kind of tuning or adjustments. In the first stage the initial validation and mesh convergence study were performed in $2 \mathrm{D}$. The 2D mesh was simple, structured and conformal, formed by hexahedral cells with a single layer in the spanwise direction. The wave generation boundary (i.e. interface with the Lagrangian model) was located at $x=20 \mathrm{~m}$ and the cells in the vicinity of that area were $5 \mathrm{~mm}$ long in the horizontal direction. Starting from $x=25.6 \mathrm{~m}$, the cell size in the wave propagation direction $(x)$ started growing progressively until $500 \mathrm{~mm}$ at the opposite end of the CFD flume, located $30 \mathrm{~m}$ away. This long distance was chosen to delay any possible reflections from the end boundary while the very coarse mesh gradation is commonly applied to dissipate wave energy numerically (Skene et al., 2018) while noticeably reducing the computational cost (as compared to using the regular cell size everywhere). The mesh was $1.1 \mathrm{~m}$ in the vertical direction, with vertical cell sizes varying from $10 \mathrm{~mm}$ to $5 \mathrm{~mm}$ from $z=0$ to $z=0.5 \mathrm{~m}$ and from 5 $\mathrm{mm}$ to $10 \mathrm{~mm}$ from $z=0.9 \mathrm{~m}$ to $z=1.1 \mathrm{~m}$. The vertical cell size remains constant through the entire length of the flume. This way a detailed resolution of $5 \mathrm{~mm}$ is obtained at the area of interest while minimising the total number of cells.

Regarding BCs, waves were generated with the coupling BC at the leftmost boundary $(x=20 \mathrm{~m})$ and absorbed with an active wave absorption BC (Higuera, 2020) at the opposite end. The bottom BC is a wall condition (no-slip) while the top $\mathrm{BC}$ corresponds to atmospheric pressure.
The lateral walls impose the desired 2D behaviour, with a free-slip BC that disables calculating the $y$-direction momentum equation. This case has also been run with RZs at the generation and absorption ends. The RZ layout used in this case spans for $20 \mathrm{~cm}$ (i.e. 40 cells) for wave generation and $20 \mathrm{~m}$ at the pure absorption end. The $2 \mathrm{D}$ mesh totals 250,000 cells and a single case of $50 \mathrm{~s}$ is completed in parallel with 4 cores $(2.5$ $\mathrm{GHz}$ ) in less than 1.5 hours.

The comparisons between the 2D CFD and the Lagrangian time series of FSE bear a high degree of accordance at the theoretical focussing point $(x=23 \mathrm{~m})$, as shown in Figure 2. The results indicate that both the BC and RZ coupling approaches produce very accurate results, nevertheless, minor differences can still be observed. For example the BC simulation produces a slightly better wave shape before the focussing event $(t=36.4$ $\mathrm{s})$, whereas the accordance of RZ simulations is slightly better after focussing ( $t=41.4 \mathrm{~s}$ ), probably due to the higher degree of absorption of the RZ at the end of the flume. Moreover, there are virtually no differences between the results with the $5 \mathrm{~mm}$ and the $10 \mathrm{~mm}$ cell resolution meshes.

The lessons learnt with this initial 2D simulations were applied to design the latter 3D mesh and case. Since accurate FSE results were obtained just $3 \mathrm{~m}$ away from the wave generation boundary, and even closer to it, the 3D mesh will start at $x=23$ m to reduce the computational cost by avoiding to simulate 3 additional metres. Moreover, since the $10 \mathrm{~mm}$ resolution has proven to be sufficiently accurate, 3D mesh will use such cell size. Finally, despite the fact that the BC simulation configuration is more computationally efficient and shows a slightly higher fidelity prior to the focussing event, both the $\mathrm{BC}$ and $\mathrm{RZ}$ techniques will be tested.

The 3D mesh has been designed to be a high quality structured cylinderfit mesh. The sketch of the mesh (top view) showing all the different blocks (red lines) used is presented in Figure 3. The overall length of the mesh is reduced with respect to the 2D case $(x=23 \mathrm{~m}$ to $x=45 \mathrm{~m})$ and the vertical dimension was set to $1.1 \mathrm{~m}(z=0 \mathrm{~m}-z=1.1 \mathrm{~m})$. The 3D mesh is $1.1 \mathrm{~m}$ in the spanwise direction $(y=0 \mathrm{~m}-y=1.1 \mathrm{~m})$, which corresponds to half of the domain of the physical flume. This reduces the computational costs by half assuming an overall symmetric behaviour of the case.

The general cell size is approximately $10 \mathrm{~mm}$ in all three directions and due to the geometry of the blocks it gets reduced to $1.5-1.8 \mathrm{~mm}$ in the horizontal directions near the cylinder wall, while the vertical cell size remains constant everywhere and equal to $10 \mathrm{~mm}$. As observed in Figure 3 certain areas closer to the lateral wall (e.g. top left corner), which are relatively far from the area of interest, present noticeably larger cell sizes in the horizontal directions. The cell size in the $x$ direction also grows larger, up to $25 \mathrm{~cm}$, near the wave absorption boundary $(x=45$ $\mathrm{m})$ to reduce the total number of cells.

The BCs are the same as in the 2D case, save the lateral boundaries, one of which is a symmetry plane. The boundary opposite to it and the cylinder surface are both walls (no-slip). The 3D mesh totals 7 million cells and a each case of $50 \mathrm{~s}$ is completed in parallel with a 72 cores $(2.5$ $\mathrm{GHz}$ ) in less than 29 hours.

Turbulent effects are expected to be very limited in time and space in the present case, and constrained to the vicinity of the cylinder right after the focussed wave crest makes impact. In view of this, all the cases have been deemed as laminar, and calculated as so. Nevertheless, the 3D case has also been run with the k- $\omega$ SST turbulence model (Devolder et al., 2017) for the BC setup, and results are reported in the next section. In both cases, the Courant number was set to 0.25 , a value that has been found low enough to provide a reasonable accuracy while keeping a good balance with the computational time required to simulate the case. 


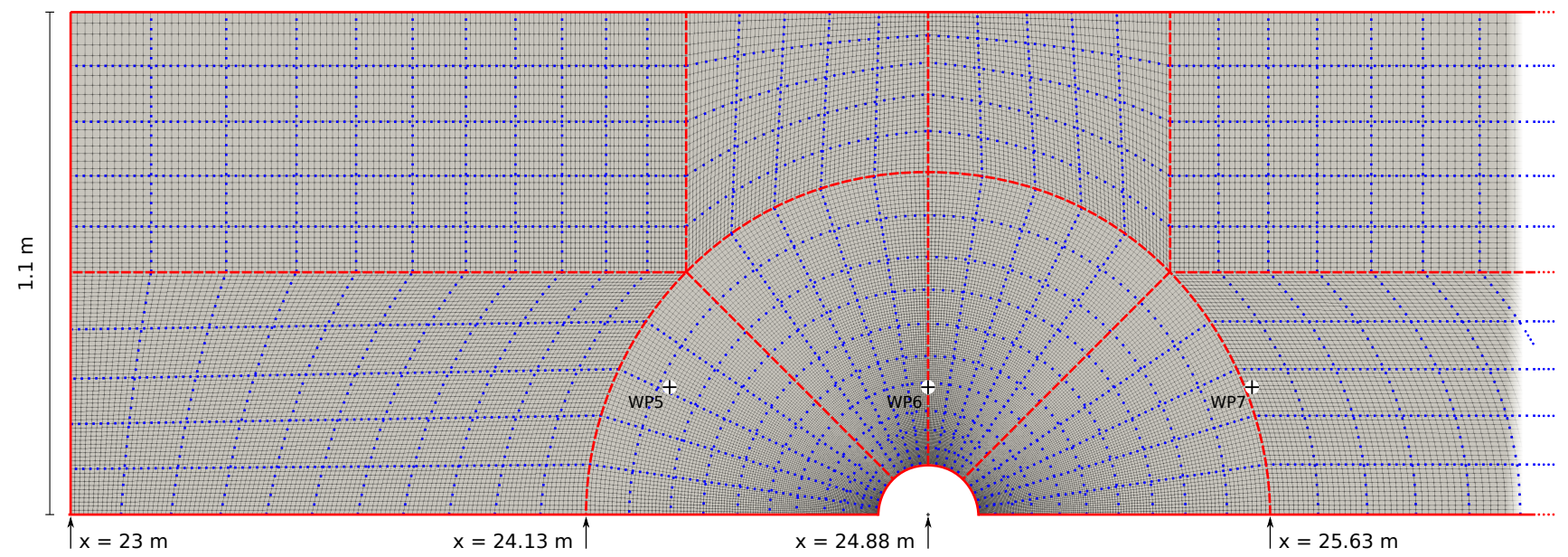

Fig. 3 olaFlow numerical mesh (top view). The mesh cells are plotted in black and the individual blocks of the mesh are outlined in red lines. Since cells might not be visible at certain locations due to image resolution, blue dotted lines indicate the internal structure of such blocks. The location of free surface elevation probes WP5-WP7 is included in the sketch.

\section{RESULTS}

The results from the free surface elevation (WP5-WP7, Table 1) and pressure gauges mounted on the cylinder surface (Table 2) are analysed in this section. It must be noted that in the case named SST the waves have been generated with the $\mathrm{BC}$ technique, and in the case named $B C$, the turbulence has not been modelled (i.e. laminar assumption).

Figure 4 presents WP5 and PP2 comparisons over the entire time series simulated in order to provide an overview of the accuracy of the 3D simulations in dealing with the waves before and after the focussing event. More detailed comparisons for the focussing event will be presented later in this section. It must be noted that since the 3D mesh starts at $x=23$ $\mathrm{m}$, the simulated time starts at the experimental time $t=10 \mathrm{~s}$, when the leading waves begin to arrive at that location.

The degree of accordance between the experimental and numerical results before focussing is appropriate and there are no evident initial discrepancies between the BC and RZ approaches. Some moderate pressure oscillations appear in the numerical solution when wave conditions are mild and are larger in the case with the k- $\omega$ SST turbulence model. These fluctuations may appear in the figure because numerical data is reported at each time step, which is variable but typically on the order of $0.0005 \mathrm{~s}(\approx 2,000 \mathrm{~Hz}$ sampling rate), whereas the experimental results (collected at 9,600 Hz) are reported at $100 \mathrm{~Hz}$ and/or may have been filtered. As mentioned before, there are some moderate noticeable differences between the BC and RZ cases starting after the wave focussing has occurred $(t>42 \mathrm{~s})$, most probably arising from the difference in the absorption performance of each method.

Figure 5 shows the FSE elevation comparisons for gauges WP5-WP7 during the focussing event. The location of the gauges relative to the cylinder can be observed in Figure 3. WP6 is located leeward from the cylinder and $1.31 \mathrm{~m}$ after the theoretical focussing location. This gauge presents the best results in terms of matching the amplitude of the focussed wave. Both BC simulations, for the laminar and k- $\omega$ SST turbulence model, capture the crest peak within an accuracy of $1.6 \mathrm{~mm}$ while the RZ simulation is $8.5 \mathrm{~mm}$ below and, therefore, the peak occurs 0.02 $\mathrm{s}$ later. The wave troughs right before and after the main wave crest are overestimated by 10 and $8 \mathrm{~mm}$, respectively, which produces a limited but noticeable difference in the wave shape, namely with a steeper front and a milder tail. Another discrepancy can be noticed at $t=40.22 \mathrm{~s}$, when a steep wave front appears in all the numerical simulations, but not in the experimental data. This is caused by the wave that is radiated at the cylinder and it propagates back after rundown. High frequency oscillations on top of the incoming waves can be observed after $t=41.7 \mathrm{~s}$ due to the reflections on the lateral boundaries.

The results at WP6, which is located side by side in line with the centre of the cylinder are very similar. The troughs are still underestimated by approximately $1 \mathrm{~cm}$, however, the numerical model now overestimates significantly the focussed wave crest, by $16 \mathrm{~mm}$ (RZ) and $33 \mathrm{~mm}(\mathrm{BC})$. The trough amplitude appears to be better captured at WP7, which is located seaward from the cylinder. However, additional 3D simulations with the inlet boundary at $x=20 \mathrm{~m}$ did not present significant improvements at WP5-WP6, indicating that the mismatch at those gauges is not caused by boundary effects because of the proximity of the wave generation boundary. The wave amplitude continues to be overestimated at WP7, ranging between $19 \mathrm{~mm}$ and $32 \mathrm{~mm}$. Nevertheless, the overall agreement and the wave shape appear to be captured better.

Figure 6 shows the pressure comparisons for probes PP1-PP8 during the focussing event. It is noteworthy that no experimental data was provided for gauge PP1. In general no significant differences can be found between all three numerical cases, except for the differences in the amplitude of the largest peak and in the latter stages $(t>41.2 \mathrm{~s})$. As expected, since the wave troughs were underestimated in terms of FSE this is also the case in terms of the numerically simulated pressures (e.g. PP2, PP3). Another general trend that is that the main pressure peaks are lower for the $\mathrm{RZ}$ case and higher for the $\mathrm{BC}$ cases, with the experimental pressure usually lying between the RZ and BC cases values (e.g. PP2-PP5) with deviations of approximately $50 \mathrm{~Pa}$ between them.

There are some gauges that are initially outside the water and measure pressure only when the FSE is higher during the course of the simulation. For example PP4 successfully captures the timing and magnitude of 4 events and PP5 does so with the single peak that reaches it. The shape of the wave in PP7 is very particular, with flat crest and troughs, because the gauge is mounted at $90^{\circ}$ with respect to the wave propagation direction.

Broadly speaking, the overall degree of accordance for pressure time series is higher than for FSE.

Figure 7 shows a snapshot of the 3D CFD simulation with several different views of the focussed wave during the impact on the cylinder, which 

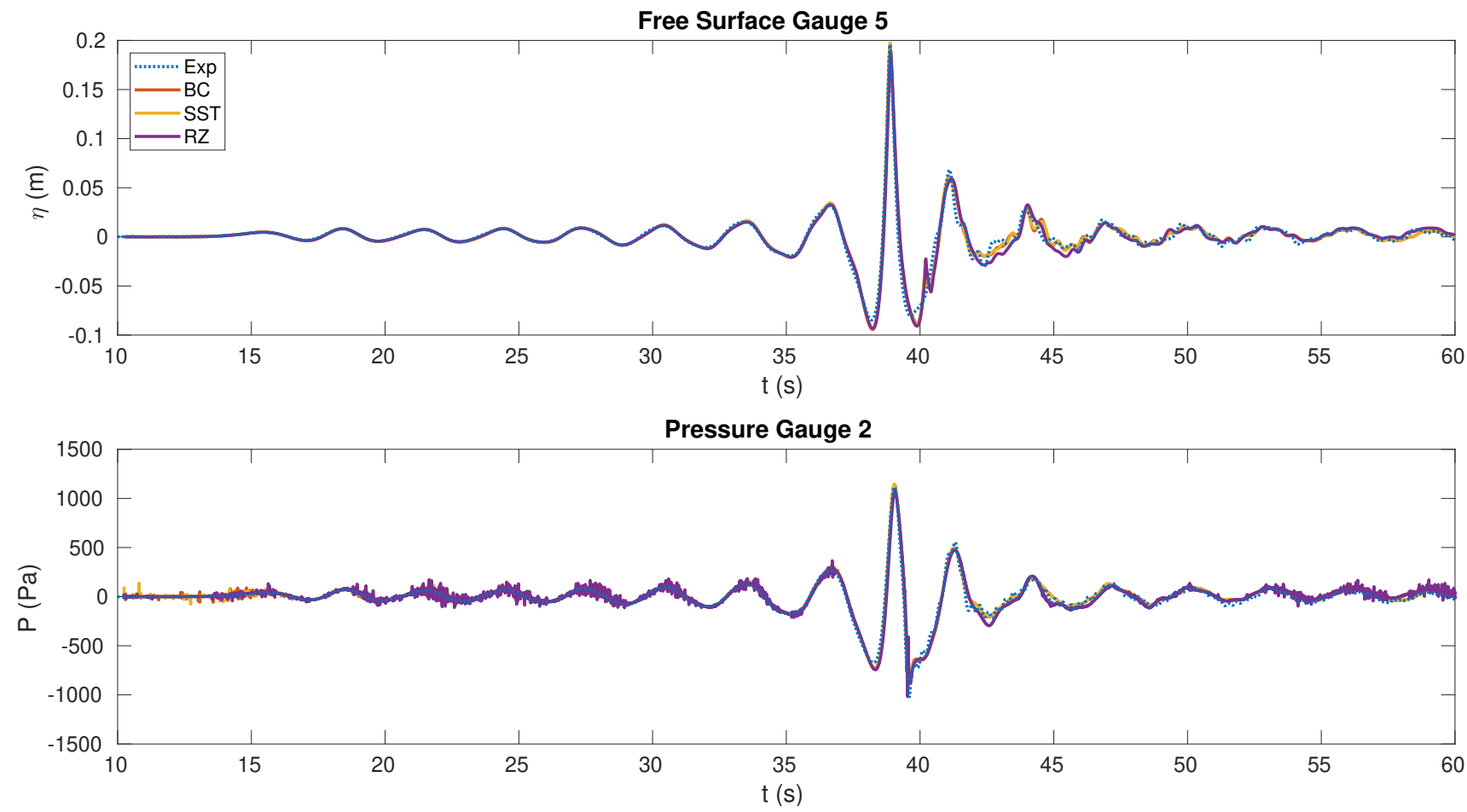

Fig. 4 Comparison of FSE and pressures between the experiments and the CFD simulations at gauges WP5 and WP2. Complete time series.
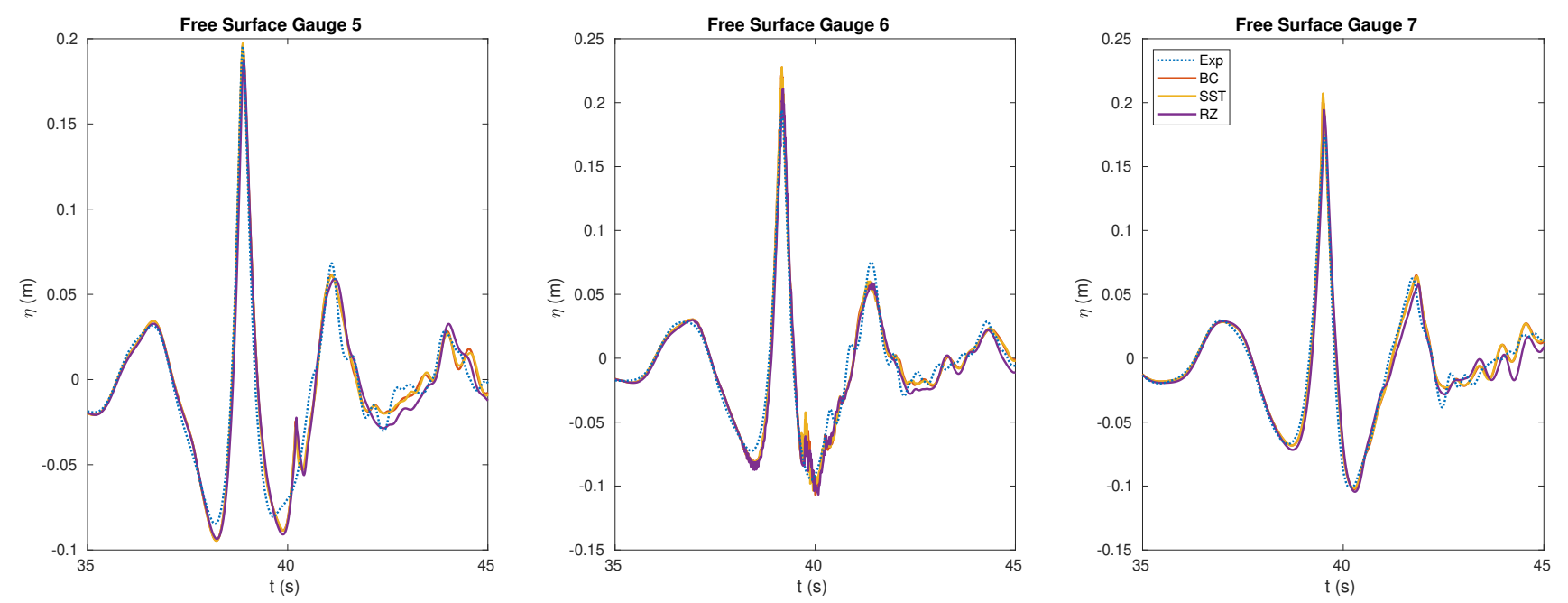

Fig. 5 Comparison of FSE between the experiments and the CFD simulations at gauges WP5-WP7. Time series in the vicinity of the focussing event.

permit observing the 3D flow features of this case. The top panel shows that the wave crest has just surpassed the cylinder and the water is wrapping around its surface and creating an enhanced runup at the back. At this instant a significant amount of water is still visible at the front part of the cylinder. Small ripple-like oscillations on the water surface can be observed around the cylinder (bottom left panel). These are most likely numerically-induced due to the cells faces at that locations not being perfectly aligned with the wave propagation direction.

\section{CONCLUDING REMARKS}

In this paper we have analysed the interaction of a focussed wave group impacting on a cylinder, modelled by a combination of a Lagrangian and a CFD models. The Lagrangian numerical model (Buldakov et al., 2019) has been proven to be faster than the CFD model and to produce very accurate wave kinematics in $2 \mathrm{D}$, which can be used as wave generation input for the CFD model closer to the structure of interest. The oneway coupling methodology between models developed in Higuera et al. (2018) has been updated as part of this work. The new coupling technique allows blending the incident wave Lagrangian kinematics over a region adjacent to the wave generation boundary with a relaxation zone (Jacobsen et al., 2012) and is complementary to the existing BC. The model olaFlow is then used to simulate the detailed wave group interaction with the fixed cylindrical structure in 3D.

Overall the degree of accordance between the experimental and numerically generated data is adequate, with mild underestimations of the wave 

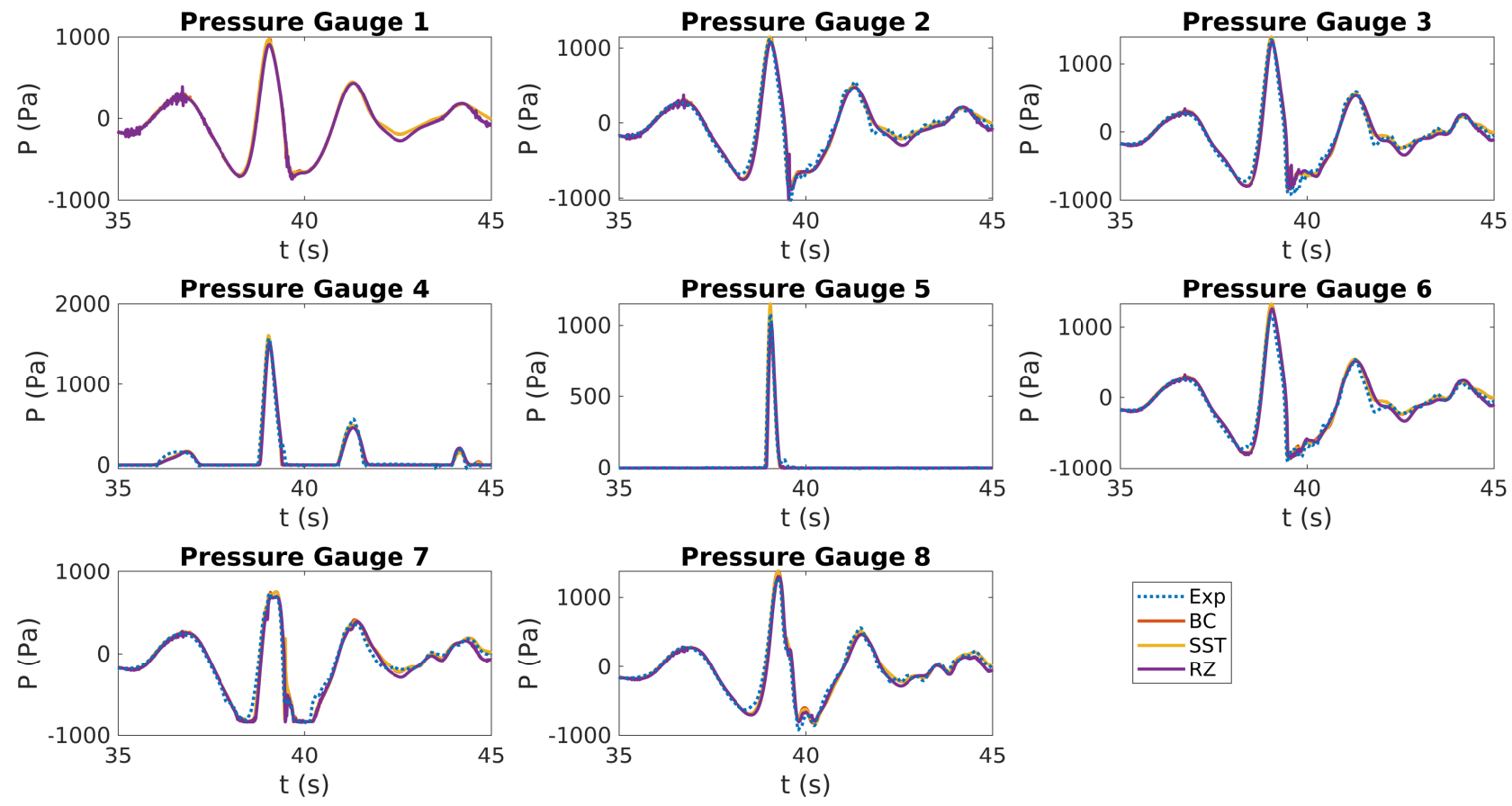

Fig. 6 Comparison of pressures between the experiments and the CFD simulations at gauges PP1-PP8. Time series in the vicinity of the focussing event.

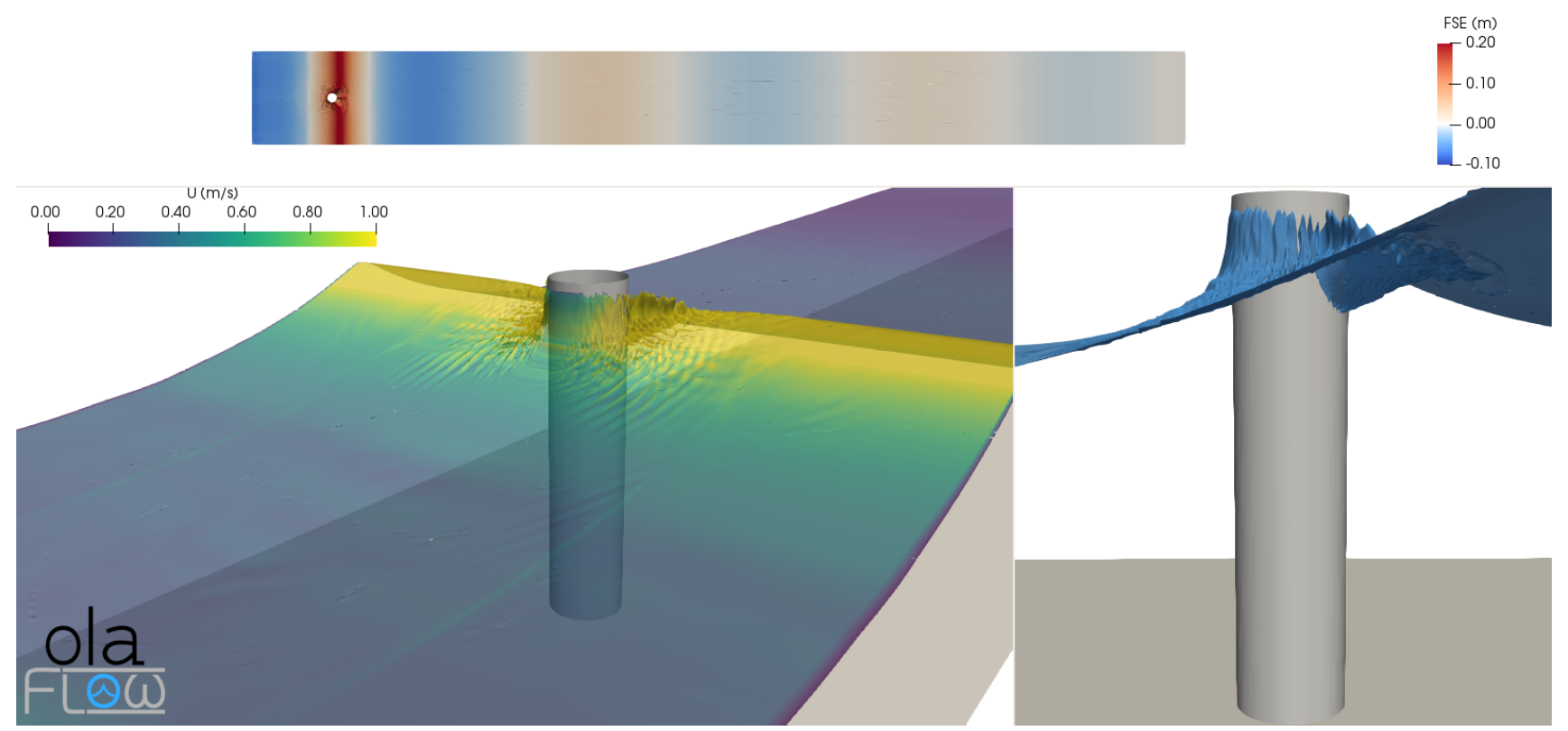

Fig. 7 3D renderings of the interaction of the focussed wave and the cylinder upon impact $(t=39 \mathrm{~s})$. FSE viewed from the top (top panel). Flow velocities at the free surface from an isometric perspective (bottom left panel). Free surface configuration from a lateral view (bottom right panel).

troughs in the vicinity of the focussed group in terms of FSE and pressures and local overestimations of FSE at the peak of the main wave. Generally the pressure time series shows a better agreement than FSE. The results also indicate that turbulence does not play a significant role in this particular test, despite the violent FSE elevation gradients measured after the wave splash and runup on the cylinder. Differences between the
$\mathrm{BC}$ and $\mathrm{RZ}$ wave generation approaches are also very limited.

In view of the results presented in this paper we have identified several improvements that will be performed as a future work. The RZ approach is promising in terms of wave absorption performance, especially regarding the benefits of linking it with active wave absorption . Furthermore, 
RZs have proven effective to generating much steeper waves than the BC method allows, thus opening a possibility to shorten the simulation domains even more by having the wave generation zone closer to the structure. However, this implementation will require guidelines to identify its best-performing parameters, namely the relaxation zone length and the shape of the blending function, including the function itself and any parameters that it might depend on (e.g., $P$ parameter). Another improvement to analyse would be the combined BC-RZ wave absorption efficiency to shorten the domain leeside the cylinder. This might not be so critical, because the number of cells in that area is decreasing due to the aggressive cell grading chosen $(1 \mathrm{~cm}$ to $25 \mathrm{~cm})$. Tightly linked to this, the number of spanwise cells in that area could be reduced progressively as we move away from the cylinder and the expected 3D effects dilute. Leaving an effectively 2D flume towards the end of the CFD domain will contribute significantly to reduce the computational cost of the 3D simulations. Finally, the ultimate long-term goal of this project would be to implement a two-way coupling between the Lagrangian and olaFlow models, thus making concurrent runs in which incoming waves would interact nonlinearly with reflections starting from the wave generation boundary, as it occurs in the experimental facility.

\section{ACKNOWLEDGMENTS}

The third author would like to acknowledge Cranfield University QR Global Challenges Research Fund (GCRF) 2019/20 for its financial contribution and support.

\section{REFERENCES}

Berberovic, E., Hinsberg, N. P. v., Jakirlic, S., Roisman, I. V., \& Tropea, C. (2009). Drop impact onto a liquid layer of finite thickness: Dynamics of the cavity evolution. Physical Review E, 79, 036306.

Buldakov, E., Higuera, P., \& Stagonas, D. (2019). Numerical models for evolution of extreme wave groups. Applied Ocean Research, 89, 128-140.

Devolder, B., Rauwoens, P., \& Troch, P. (2017). Application of a buoyancy-modified k-omega SST turbulence model to simulate wave run-up around a monopile subjected to regular waves using OpenFOAM. Coastal Engineering, 125, 81-94.

Fuhrman, D. R., Madsen, P. A., \& Bingham, H. B. (2006). Numerical simulation of lowest-order short-crested wave instabilities. Journal of Fluid Mechanics, 563, 415-441.
Guo, L.-D., Sun, D.-P., \& Wu, H. (2012). A new numerical wave flume combining the 0-1 type BEM and the VOF method. Journal of Hydrodynamics, Ser. B, 24(4), 506-517.

Higuera, P. (2017). olaflow: CFD for waves. URL https://doi .org/10.5281/zenodo. 1297013

Higuera, P. (2020). Enhancing active wave absorption in RANS models. Applied Coastal Research, 94.

Higuera, P., Buldakov, E., \& Stagonas, D. (2018). Numerical modelling of wave interaction with an FPSO using a combination of OpenFOAM and Lagrangian models. In Proceedings of the 28th International Ocean and Polar Engineering Conference, June 10-15 2018, Sapporo, Japan.

Higuera, P., Lara, J. L., \& Losada, I. J. (2013). Realistic wave generation and active wave absorption for Navier-Stokes models: Application to OpenFOAM. Coastal Engineering, 71, 102-118.

Jacobsen, N. G., Fuhrman, D. R., \& Fredsøe, J. (2012). A wave generation toolbox for the open-source CFD library: OpenFOAM. International Journal for Numerical Methods in Fluids., 70(9), 10731088.

Kim, S.-H., Yamashiro, M., \& Yoshida, A. (2010). A simple twoway coupling method of BEM and VOF model for random wave calculations. Coastal Engineering, 57(11), 1018-1028.

Lachaume, C., Biausser, B., Grilli, S., Fraunié, P., \& Guignard, S. (2003). Modeling of breaking and post-breaking waves on slopes by coupling of BEM and VOF methods. In Proceedings of the Thirteenth International Offshore and Polar Engineering Conference, (pp. 1698-1704). Honolulu, Hawaii, USA.

Schäffer, H. A. (1993). Second order irregular-wave generation in flumes-computation of transfer functions by an asymptotic summation method. In Ocean Wave Measurement and Analysis, (pp. 784797). ASCE.

Skene, D. M., Bennetts, L. G., Wright, M., Meylan, M. H., \& Maki, K. J. (2018). Water wave overwash of a step. Journal of Fluid Mechanics, 839, 293-312.

Sriram, V., Schlurmann, T., \& Schimmels, S. (2015). Focused wave evolution using linear and second order wavemaker theory. Applied Ocean Research, 53, $279-296$.

Weller, H., Tabor, G., Jasak, H., \& Fureby, C. (1998). A tensorial approach to computational continuum mechanics using objectoriented techniques. Computers in Physics, 12(6), 620-631. 